\title{
Sleep Apnoea and the Brain: A Complex Relationship
}

\author{
Ivana Rosenzweig ${ }^{1,2,3}$, Martin Glasser ${ }^{4}$, Dora Polsek ${ }^{1,5}$, Guy D. Leschziner ${ }^{1,3,6,7}$, Steve C.R. \\ Williams $^{1}$, Mary J. Morrell ${ }^{1,4}$
}

\footnotetext{
${ }^{1}$ Sleep and Brain Plasticity Centre, Department of Neuroimaging, IOPPN, King's College and Imperial College London, United Kingdom.

${ }^{2}$ Danish Epilepsy Centre, Dianalund, Denmark.

${ }^{3}$ Sleep Disorders Centre, Guy's and St Thomas' NHS Foundation Trust, UK.

${ }^{4}$ Academic Unit of Sleep and Breathing, National Heart and Lung Institute, Imperial College London, UK and NIHR Respiratory Disease Biomedical Research Unit at the Royal Brompton and Harefield NHS Foundation Trust and Imperial College London.

${ }^{5}$ Croatian Institute for Brain Research, University of Zagreb School of Medicine, Croatia.

${ }^{6}$ Department of Neurology, Guy's and St Thomas' NHS Foundation Trust, UK.

${ }^{7}$ Department of Clinical Neuroscience, IOPPN, King's College London, London, UK
}

Word Count: 5401 words

References: 106

4 Illustrations

Corresponding author: Dr Ivana Rosenzweig, Department of Neuroimaging, Box 089, Institute of Psychiatry, Psychology and Neuroscience (IOPPN), De Crespigny Park, King's College London, SE5 8AF, United Kingdom. Phone: + 44203228 3084. Email: ivana1.rosenzweig@kcl.ac.uk .

Acknowledgments: This work was supported by the Wellcome Trust [103952/Z/14/Z] and the NIHR Respiratory Biomedical Research Unit at the Royal Brompton and Harefield NHS Foundation Trust, Imperial College London.

Contributors: All authors contributed equally to the writing and revision of this manuscript.

The authors do not have any conflicts of interest to report. The authors apologize to all the colleagues whose outstanding work could not be cited due to space limitations. 


\section{Summary (151/150 words)}

The complex relationship between obstructive sleep apnoea (OSA) and the brain is explored in this review. During the untreated apnoea/hypopnoea, intermittent hypoxia, reoxygenation and hyper/hypocapnia occur, in both adults and children, along with changes in cerebral blood flow and sleep fragmentation. They may in time result in cognitive deficits with functional consequences for work and school efficiency.

The ability to assess how OSA affects cognition depends on the specificity and sensitivity of the tests, which are rarely developed specifically for OSA. In this review we discuss both the neural adaptive and maladaptive processes to hypoxemia. Their net result on cognitive and emotional performance depends on the stage of this dynamic process, effects on other body systems, cognitive reserve and the idiosyncratic susceptibility. The contribution of fragmented sleep and the disruption of sleep structure is also explored, with a focus on its effects at different times in the genesis of the disease. 


\section{Introduction}

Obstructive sleep apnea (OSA) is a highly prevalent, chronic multisystem disease that remains under recognised in the general population, despite the 20 years that have elapsed since the seminal prevalence report was published in 1994, and over 30 years since the acclaimed account of

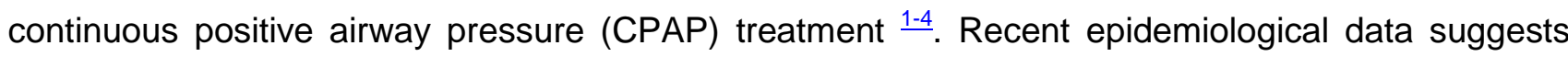
that there has been a $14-55 \%$ increase in the prevalence of OSA in the USA since the early $1990 \mathrm{~s} .{ }^{5}$ Similar prevalence distribution figures have been noted in other countries and specific ethnic groups around the world, with clear overall male preponderance until menopause age in women. $\frac{6-10}{\text { OSA is }}$ commonly associated with serious cardiovascular and metabolic co-morbidities, with systemic hypertension occuring in up to $50 \%$ of OSA patients. $\stackrel{11}{12}$ Its strong associations with old-age and obesity, both of which are on the rise, underscore its important current and future public health implications $\underline{11}, \underline{13}$.

The syndromic constellation of effects of OSA on the brain merit particular attention and are the focus of this review. OSA is increasingly recognised as one of the potentially modifiable risk factors for dementia, especially in older adults ${ }^{14-16}$. Moreover, its role in seizure aggravation in epilepsy,

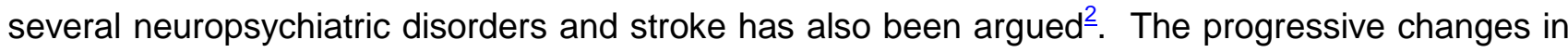
sleep quality and structure, along with changes in the cerebral blood flow, neurovascular and neurotransmitter changes, the cellular redox status and neural regulation in OSA patients may constitute a contributing factor to cognitive decline, also seen with healthy aging, but present to a much greater extent in neurodegenerative diseases 1 , 16-19.

Reduced quality of life, excessive daytime sleepiness, labile interpersonal relationships, increased road traffic accidents and decreased work and school efficiency have all been reported in OSA

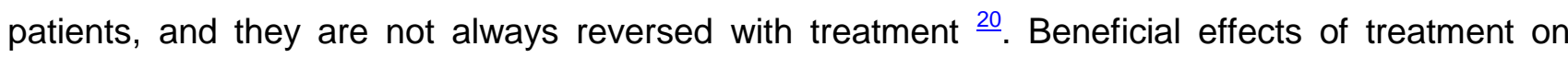
cognitive performance (including vigilance and sleepiness) and neural injury in OSA (Figure 1) are,

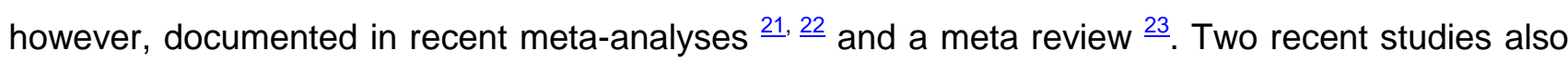
suggest beneficial effects of the CPAP therapy in minimally symptomatic, and older OSA patients, respectively $\underline{24}, \underline{25}$. The current dearth of fully effective treatments for the central nervous system sequelae of OSA is likely to be a reflection of, as yet poorly understood, intricate interplay of intermittent hypoxia, reoxygenation, hypercapnia, changes in cerebral blood flow $\underline{26}$, and sleep fragmentation that results from nocturnal episodes of complete or partial pharyngeal obstruction in $\operatorname{OSA}^{1}, \underline{27}$.

In recent years, we and others have argued that both adaptive and maladaptive processes to hypoxemia occur in brains of OSA patients. Their overall net result depends on the stage of this dynamic process, effects on other body systems, cognitive reserve and the idiosyncratic susceptibility of each particular patient (for in depth reviews please refer to $\underline{2}, \underline{15}, \underline{17}, \underline{27-31}$ ). This is important as different therapeutical approaches might benefit different stages and conversely might 
aggravate damage in some patients $\underline{2}, \underline{17}, \underline{28}$. To date, the contribution of fragmented sleep to cognitive and emotional performance of OSA patients has been largely overlooked ${ }^{2}$. Also, the diversity of published studies make it difficult to delineate the impact of its interplay with ongoing

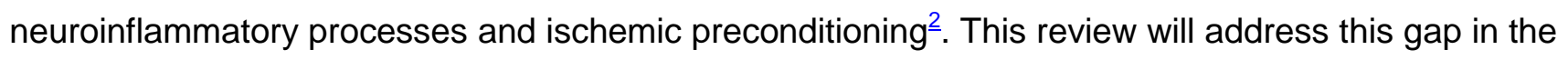
background of new clinical and translational findings, and to argue their contribution to the inherent complexity of the relationship between OSA and the brain.

\section{The myth and reality of impaired cognition in patients with sleep apnoea}

A broad range of psychological problems and neurocognitive impairments have over the years been more or less consistently associated with $\mathrm{OSA}^{\underline{23}}$. Despite this fact, it has been recently argued that detailed analysis of important studies in the field allows for only a weak correlation between (subjective) cognitive complaints in patients with OSA and their objective cognitive functioning $\underline{32}$. This argument has already been used to highlight divergent results of subjective versus objective complaints in other medical populations, and several possible explanations for this in OSA patients have been suggested. For example, an insufficient specificity of current tests for deficits documented in OSA is evident and largely acknowledged. Currently used and validated objective tests for cognition are frequently designed to cater for deficits found in brain-injured patients and as such do not specifically assess impairments in OSA ${ }^{32}$. Similarly, it is accepted that testing provides only a snapshot of someone's ability, and that it is unlikely that it accurately assesses those aspects of cognition of patients in OSA that are task dependent or time dependent. Hence it follows that, given the complex make up of neuropathology of OSA, it is likely that the snapshot value of a particular function or ability, assessed by cognitive tests, does not fully account for its fluctuation over time and various daytime requirements. Cognitive domains are not unitary constructs, and only the carefully deconstructed analysis of their different subcapacities and their vulnerabilities to a range of risks and protective factors specific to OSA can provide a more realistic assessment of someone's deficits ${ }^{22}$. Similarly, a number of impairments may be secondary to other symptoms of OSA, such as sleepiness, or they can be a sign of psychological distress $\underline{32}, \underline{33}$.

Of note, non-pharmacological and some pharmacological treatments for OSA have been shown to improve cognitive outcomes in OSA patient subpopulations. Several meta-analyses suggest that CPAP treatment reduces sleepiness complaints and mood problems, and that it improves objective cognitive functioning in OSA patients $\mathbf{2 1}^{1}, \underline{32}, \underline{34}, \underline{35}$. Many questions regarding treatment with CPAP, most pivotal of which are who, when and for how long should receive this treatment, are still unclear. The full prescriptive protocols, likely in combination with other lifestyle and/or pharmacological approaches, might only be achieved once the full spectrum of neuropathology of OSA and its dynamic fingerprinting are understood. ${ }^{2}, \underline{27}$ For example even that the beneficial effect of CPAP on symptoms of sleepiness and quality of life in some patients appear to be obtained after only few days of treatment with a good adherence, the effects on other subjective and objective 
cognitive symptoms are less well defined. $\underline{36}, \underline{37}$ Two recent studies suggest that prolonged treatment might be required in some severe patients. $\frac{38}{39}$ An almost complete recovery of white matter tracts pathology with associated significant improvement in memory, attention and executive functioning has been demonstrated in a recent study only following one year of a compliant treatment in severe OSA patients. ${ }^{38}$ The need for longer treatment with CPAP in elderly has also been suggested by findings of another small pilot study which has shown that treatment of severe OSA in Alzheimer's patients (of mild to moderate severity) is associated with significantly slower cognitive decline over three years. ${ }^{39}$ Although less striking, limited evidence with drugs such as donepezil, physiostigmine, and fluticasone also points to better cognitive outcomes in treated patients ${ }^{40}, \underline{41}$. So far, subjective cognitive complaints have been largely ignored in randomised controlled trials of treatments for OSA patients. In line with recent findings, and given that subjective cognitive complaints are known to be linked to quality of life, work productivity and health care utilisation in the general population future studies should account for them $\underline{32}$.

Some authors have also argued against a reductionist approach to OSA-induced brain injury, and point out that emerging research indicates that the relationship between disease severity and cognitive dysfunction is the product of a multitude of susceptibility and protective factors, of which sleep fragmentation, hypoxia and cognitive reserve are only three aspects $\underline{2}, \underline{22}, \underline{27}$. Some other, commonly ignored, important factors are duration of disease, the role of the blood-brain barrier, hypertension, metabolic dysfunction, systemic inflammation, cerebral blood flow, and genetic vulnerability (e.g. apolipoprotein e4 genotype) $)^{\underline{22}}$.

In the face of ongoing polemics in the field, the majority of studies to date nonetheless agree on significant deficits in attention/vigilance, delayed long-term visual and verbal memory, visuospatial/constructional abilities and executive dysfunction in patients with $\mathrm{OSA}^{\underline{23}}$, $\underline{42}$. Several associations have been recognised: the association between the worsening in global cognitive functioning and the severity of hypoxaemia, and the association between the attention and vigilance dysfunction and the degree of sleep fragmentation $\underline{23}$. Consensus in the field is less strong on the effects of OSA on working memory and short-term memory $\underline{23}$. Also, language ability and psychomotor functioning have been shown to be largely unaffected by $\mathrm{OSA}^{\underline{23}}$ in some studies, whilst others have pointed to psychomotor slowing as the most vulnerable cognitive domains, and least responsive to the treatment with $\mathrm{CPAP}^{43}$. Similarly, several studies that have shown impairments in language abilities in severe OSA patients have disagreed on whether phonemic or semantic domains are those of highest impact. ${ }^{44}$. Neurodevelopmental stages of adolescents and children with OSA appear to dictate higher risk for this deficit $\frac{45}{5}$.

The functional neuroanatomy of OSA has been further highlighted in a study following 3 months of treatment with CPAP that demonstrated improved cognitive function in several domains that corresponded to grey matter volume increases in frontal and hippocampal regions $\frac{46}{}$. Similarly, 
another recent study has shown significant improvements involving memory, attention, and executive functioning that correlated to the white matter changes after 12 months of treatment with $\mathrm{CPAP}^{38}$. The majority of studies investigating treatments for OSA failed to account for incomplete reversal of reversal of tissue damage, or deficits in cognition, suggesting that initiation of a (prolonged) treatment might be needed as early as possible in disease process ${ }^{47-49}$.

In children, perhaps unsurprisingly, the results of studies looking into the cognitive performance and effects of treatment in children with OSA are equally divergent $\underline{50,51}$. In a recent study of children with sleep disordered breathing (SDB), followed for four years, treatment led to improvements in several aspects of neurocognition, collectively categorised as performance IQ ${ }^{50}$. Performance IQ represents fluid intelligence that is reflective of incidental learning, and it describes one's ability to adapt to new situations ${ }^{\underline{52}}$. In this study, improvements were recorded in tasks associated with spatial visualisation, visuo-motor coordination, abstract thought and nonverbal fluid reasoning $\underline{50}$. However, overall improvements in the academic ability or behaviour were less clear. Furthermore, worsening of verbal IQ, which, unlike performance $I Q$, is more likely to be affected by formal education and learning experiences, was noted in a treated group ${ }^{50}$. A definitive explanation for this finding was not provided, and no statistically significant association between the reduction in verbal IQ performance and treatment was demonstrated ${ }^{50}$. Conversely, another influential study where younger children with SDB were followed for 12 months of treatment, showed significant improvements in academic performance $\frac{51}{}$. The different neurodevelopmental ages of children, and different test parameters used, provide for a complex clinical data set, against which no finite conclusions can be drawn. Nonetheless, particular patterns and associations seem to be emerging from this and earlier work, amongst which the association between performance IQ and slow wave activity (SWA) during the non-rapid eye movement (nREM) sleep is perhaps the strongest one ${ }^{50}, \underline{53}$. In healthy adults, sleep progresses through nREM stages N1 through N3 followed by a period of REM sleep occurring approximately 60- 90 minutes into sleep ${ }^{54}$. It has been argued that cognitive improvements in treated OSA patients may reflect increased stability of brain activity during sleep, allowing for a crucial synaptic repair/maintenance to occur and counteracting toxic effects of arousal and hypoxic effects of OSA $\underline{50}, \underline{55}$. This argument is concordant with findings showing that the neurochemical and gene environments of sleep and sleep activity patterns present crucial window periods during which the brain can restore cellular homeostasis, increase signal to noise ratio, and reinforce neuronal circuitry for subsequent cognitive processing demands $\underline{18}, \underline{56}, \underline{57}$.

\section{3. "Cognitive and emotional" sleep of sleep apnoea}

Sleep and sleep deprivation alter molecular signalling pathways that regulate synaptic strength, plasticity-related gene expression and protein translation in a bidirectional manner $\underline{57}$. Moreover, sleep deprivation can impair neuronal excitability, decrease myelination and lead to cellular 
oxidative stress and misfolding of cellular proteins $\underline{57}, \underline{58}$. The correlations between oxidative stress markers, sleepiness and the presence of affective symptoms in patients with sleep apnoea have been suggested by findings of an open, cross-sectional, and comparative clinical trial. ${ }^{59}$ Frequent brief awakenings, and ensuing fragmented sleep, have been shown to impact next day's cognitive and emotional functioning, in a manner similar to that of total sleep deprivation ${ }^{15}$. Several studies have also attempted to assess if OSA patients were more vulnerable to sleep-loss induced performance deficits, with special emphasis on driving performance variables, with varied results $\underline{60-63}$ From the practical point of view it is of major interest to develop reliable and practical bedside tests to help clinicians advise patients on their individual traffic accidents risks. A recent preliminary study has shown potentially promising data for a specific EEG biomarker of neurobehavioural impairment and sleepiness (also see ${ }^{\underline{61}}$ ). Preclinical studies suggest that sleep fragmentation independently affects similar brain regions to those affected by the intermittent hypoxia ${ }^{1}$. Also, clinical studies of the effects of sleep deprivation on cognition in the general population suggest comparable cognitive impairments to those seen in OSA $\underline{64}$. Frequent partial arousals during sleep in OSA patients contribute to abnormal sleep architecture and symptoms of excessive daytime somnolence ${ }^{1}, \underline{27}$. An independent association between excessive daytime somnolence and cognitive impairment has been demonstrated and several prospective studies have shown that excessive daytime somnolence is associated with an increased risk of cognitive decline and dementia ${ }^{14}$. In a prospective cohort study (The Honolulu-Asia Aging Study), lower nocturnal oxygenation and reduction in SWS nREM sleep were associated with the development of microinfarcts and brain atrophy ${ }^{65}$. Conversely, men with longer SWA sleep showed slower cognitive decline $e^{\underline{65}}$.

\subsection{Cognitive sleep}

The relationship between OSA and its effect on selected sleep stages merits particular attention, given that each of the sleep stages with its attendant alterations in neurophysiology is associated with facilitation of important functional learning and memory processes $\frac{18}{}$. In OSA patients, the proportion of stage N2 nREM sleep has been shown to increase, while proportions of stages N1,

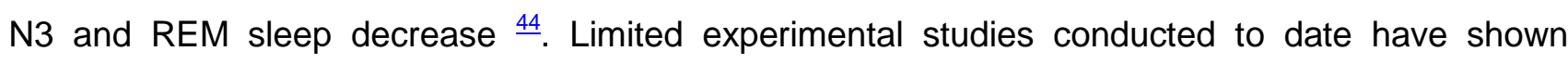
specific impairments of sleep-dependent consolidation for verbal declarative information in patients with $\mathrm{OSA}^{\underline{66}}$. Furthermore, several recent clinical studies suggest disturbed spatiotemporal evolution of sleep spindles in patients with OSA during the night $\underline{67}, \underline{68}$.

However, consequences of sleep fragmentation on sleep in OSA are not easy to assess, and dynamic analysis of sleep architecture is required in order to fully gauge the impact on sleep. For example, in one study of mild OSA, where sleep fragmentation was likely not of sufficient size to provide significant SWS (e.g. in N3 nREM) and REM sleep decreases, the exponential decay function of SWA was demonstrated as significantly slower in patients compared to controls ${ }^{\underline{69}}$. This was due to the more even distribution of SWA throughout the night. These results show that mild 
sleep fragmentation can alter the dynamics of SWA, without decreasing significantly the amounts of SWS and REM sleep and emphasize the need to perform SWA decay analysis in sleep fragmentation disorders ${ }^{69}$. In the same study, a decrease of spindle activity was observed in N2 and N3 which was not attributed to an increase of SWA $\underline{67}, \underline{69}$. Such a reduction in total spindle density has also been reported in sleep maintenance insomnia, and is likely to be related to sleep fragmentation. $\frac{67-69}{}$

More recently, an integrative framework for qualitative reorganisation of memory during sleep has been suggested ${ }^{70}$. The model proposed by Landmann and colleagues (2014) further builds on studies that have shown that sleep facilitates the abstraction of rules and the integration of knowledge into existing schemas during the nREM SWS $\underline{56}, \underline{57}, \underline{70}$. REM sleep, on the other hand, benefits creativity that requires the disintegration of existing patterns ${ }^{70}$. Of note, both sleep stages are commonly reported as reduced/fragmented in OSA, and it is likely that some of the cognitive complaints and memory deficits described are partly acquired via this dysregulation. $\underline{44}, \underline{71}$ In line with this argument, in one study that looked into the neurocognitive deficits in OSA, the number of microarousals during the night was shown as the best predictor of episodic memory deficit $\underline{72}$.

Traditionally, obstructive events during nREM sleep have been viewed as associated with greater cognitive deficits or impaired quality of life, whilst REM sleep events have been shown to be associated with greater sympathetic activity, hypertension and cardiovascular instability in patients with OSA $\underline{73} \underline{74}$. Recently, the role for fragmented REM sleep in spatial navigational memory in OSA patients has been addressed with a physiologically relevant stimulus $\underline{75}$. During this study, patients spent two different nights in the laboratory, during which they performed timed trials, before and after sleep, on one of two unique 3D spatial mazes ${ }^{75}$. The normal consolidation of sleep was achieved with use of therapeutic CPAP throughout the first night, whereas during the second night CPAP was reduced only during the REM stages. Here, patients showed improvements in maze performance after a night of normal sleep, and those improvements were significantly reduced following a night of REM disruption without changes in psychomotor vigilance. Noted cognitive improvements were significantly positively correlated with the mean REM run duration across both sleep conditions $\underline{75}$.

Another recent model might be of interest from perspective of OSA-induced sleep deficits ${ }^{54}$. Here, it is proposed that, during SWA of nREM sleep, recovery of functionally interconnected networks occurs $^{54}$. This model also stresses the importance of the appropriate spatiotemporal revolution of sleep spindles throughout nREM sleep ${ }^{54}$. Sleep spindles are taken to reflect tagging of those networks that have presumably obtained the necessary recovery for their inclusion in the selection process during subsequent sleep ${ }^{54}$. In turn, further excursions into REM sleep then allow for a safe "offline" reactivation ${ }^{54}$. This serves to check and to deselect fully recovered brain networks from further SWA cycles. Once the network specific and function dictated number of SWA cycles is 
achieved, a presumed transition to wake occurs ${ }^{54}$. Regarding OSA-induced deficits, it could be argued that this model offers robust theoretical framework for the origins of the sense of excessive daytime sleepiness and of feeling unrefreshed in the morning by some OSA patients. According to this model, this might be due to the inability to augment nREM SWA and or REM sleep during the period of high waking demands. Moreover, in some OSA patients, reduction of REM sleep can lead to dissociation of REM traits to other sleep stages, further impacting on critical sleep windows for memory formation and consolidation $\frac{18}{}$. Of particular note, several studies have now demonstrated that, if high homeostatic demands are not fully met during sleep, in the subsequent wake period, microsleeps can occur in highly active regions of the brain ${ }^{54}$. This can lead to concomitant disability for the function catered by that region $\underline{54}, \underline{56}$. To which level this takes place in OSA patients, and whether this also contributes to attention/vigilance dysfunction and higher levels of traffic accidents noted for this patient group, is yet to be fully understood. Previously reported retarded SWA decay throughout the night in even mild OSA patients further supports the notion of non-restorative sleep in OSA $\underline{69}$.

Most recently, it has been demonstrated that the degree of atrophy in the medial prefrontal cortex (mPFC), the area also known to be independently affected by OSA (Figure 1) and known to be involved in the generation of slow waves, predicts the extent of impaired SWA in the elderly, and consequent impaired overnight episodic hippocampal memory consolidation (Figure $2 a)^{\underline{19}}, \underline{57}$. It has been proposed that, at a clinical level, improving SWA in older adults may represent a novel treatment for minimising cognitive decline in later life ${ }^{19}$. Similarly, it has been recently shown that older adults who express fewer prefrontal fast sleep spindles than younger adults, also exhibit a proportional impairment in hippocampal functioning during the subsequent wake periods, and with that impairment, a deficit in the ability to form new episodic memories ${ }^{76}$. Fast sleep spindles represent part of a coordinated nREM sleep-dependent memory mechanism, and it is thought that hippocampal sharp-wave ripples provide feedback excitation, which initiates neuroplasticity in spindle-activated cortical neurons ${ }^{57}$. Relative to slow sleep spindles, fast sleep spindle events are associated with greater hippocampal activation and greater hippocampal-cortical functional connectivity $\underline{15}, \underline{76}$. Intriguingly, in a study of spindle frequency changes in OSA, it has been shown that OSA patients, unlike healthy controls, persisted in displaying a significant proportion of slow spindles in frontal, central and parietal regions during the night, perhaps suggesting that deregulated spindle formation might be another contributory facet to cognitive complaints in OSA patients ${ }^{67}$. In another study, the sleep architecture of mild OSA patients showed a high degree of sleep fragmentation resulting in a different time course of SWA and a decreased sleep spindle index when compared to controls ${ }^{69}$. Taken together, these studies also further highlight the possible role for OSA brain injury in the acceleration, or even initiation, of cognitive decline (Figure 2a) in older adults, although the exact pathophysiology of this association remains elusive $\mathrm{e}^{14-16}, \underline{77}$. 


\subsection{Emotional sleep}

A bidirectional relationship between sleep and emotional brain function is increasingly supported by findings of studies that further build on long-standing clinical observations of co-occurring mood and sleep disorders ${ }^{78}$. Perhaps then unsurprisingly, affective disorders, emotional lability and depression have all been reported as highly prevalent in individuals with OSA, with some studies reporting that up to $63 \%$ of individuals are affected despite considerable heterogeneity and a high risk of bias ${ }^{2}, \underline{79}$, 80. Evidence from various studies is particularly suggestive of role for REM sleep in selective emotional memory processing and sleep-dependent emotional memory depotentiation (Figure $2 b)^{78}$. Moreover, REM sleep is suggested to play a role in recalibrating the sensitivity and specificity of the brain's response to emotional events on both ends of positive and negative spectrum $\underline{\underline{78}}$. This effect likely occurs at least partly as a results of modulation of noradrenergic brain stem activity and the responsive profiles of the amygdala and medial prefrontal cortex (mPFC), two regions critically involved in detecting emotional salience $\frac{78}{}$.

Of all psychiatric disorders, the evidence for increased prevalence of OSA is particularly strong for

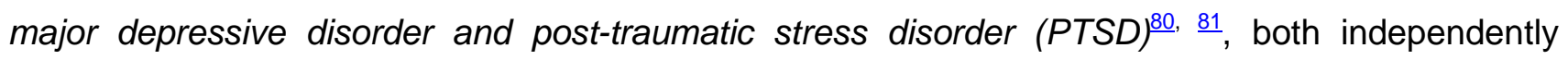
associated with REM sleep disturbance. Even though the causal relationship between these affective disorders and OSA is unclear and is likely to be multifactorial, the potential sleep mechanics of their interaction is worthy of further consideration. PTSD is independently associated with decreases in the total time spent in REM sleep. It is also associated with marked fragmentation of REM sleep, indicative of arousal-related awakenings from REM sleep linked to adrenergic surges $^{78}$. CPAP adherence has been shown to be reduced in veterans with PTSD and comorbid $\mathrm{OSA}^{80}$. Based on the current knowledge of OSA-induced sleep deficits, it can be argued that in PTSD patients with co-morbid OSA, the additive effect of sleep disturbance can further impair the quantity and quality of REM sleep in affected individuals. This would likely also impact on REM's noradrenergic "housekeeping" function, as it has been shown that REM sleep reduces, and thus likely restores, concentrations of noradrenaline to baseline, allowing for optimal wakeful functioning ${ }^{78}$. More specifically, several studies suggest that quiescence of locus coeruleus activity, a brain stem structure and a source of noradrenergic input, during REM sleep throughout the night restores the appropriate next-day tonic/phasic response specificity within emotional salience network (e.g. locus coeruleus, amygdala, mPFC) ${ }^{78}$. It is hence feasible that OSA induced REM fragmentation could further aggravate the hyperadrenergic state of some PTSD patients, and lead to decreased connectivity between the PFC and amygdala, and exaggerated amygdala reactivity ${ }^{78}$. The functional outcome may be an aggravated disease course and worse prognosis ${ }^{78}$. Of note, in a prospective study higher nocturnal oxygenation during REM sleep was associated with less gliosis and neuronal loss in the locus coeruleus ${ }^{65}$. Major depression, on the other hand, is associated with exaggerated REM sleep qualities and deficiency in monoamine activity $\underline{78}$. The dual relationship 
between two disorders has been suggested by findings of several studies ${ }^{\underline{77}}$. Arguably, in some OSA patients fragmented REM sleep can precipitate a vicious cycle of impaired regulation and rebound REM augmentation. This, along with concomitant changes in neurotransmitter systems caused by hypoxemia, could further lead to reduced monoamine activity, increased negative rumination and ensuing depression in genetically predisposed individuals. Through its effects on REM sleep, comorbid OSA might also lead to dysfunctional consolidation and depotentiation of emotional memory from prior affective experiences $\underline{78}$. It has been proposed that this may result in a condition of chronic anxiety within autobiographical memory networks (Figure $2 b)^{78}$. In recent meta-analysis of randomized controlled trials of treatment of OSA, a significant improvement in depressive symptoms was reported $\underline{79}$.

Even though all above argued theoretical constructs of a bidirectional relationship between sleep in OSA and psychiatric disorders are indirectly strongly supported by animal and neuroimaging studies of sleep (also $\mathrm{see}^{\frac{78}{}}$ ), their true mechanics are likely to be more complex.

\section{Neuroinflammation and ischaemic conditioning are two "Janus" faces of intermittent hypoxia}

Cognitive and emotional complaints of OSA patients are equally well explained by oxidative and neuroinflammatory effects of OSA on the emotional salience network $\stackrel{2}{2} \underline{27}, \underline{77}$. In OSA, repetitive occlusions of the upper airway lead to intermittent hypoxia and recurrent hypoxemia, typically characterized by short cycles of hypoxia and reoxygenation ${ }^{28}$. However, the patterns vary greatly across patients, and, depending on the idiosyncratic characteristics of each individual, the end results might be either adaptive or maladaptive ${ }^{28}$. More specifically, the outcome will also likely depend on the dynamic interplay between the specific type and amount of reactive oxygen/nitrogen species produced, their duration and frequency, the intracellular localization, and the microenvironmental antioxidant activity ${ }^{17}$. Additional interplay depends on factors such as the genetic makeup, nutrition and other lifestyle-related variables, all of which affect the redox status (also se $e^{17}, \underline{28}$ ). A variety of studies to date suggest that the severity of hypoxia, its duration, and cycle frequency, are fundamental determinants of outcomes (Figure $3 a)^{82}$. For example, it has been generally acknowledged that short, mild, and lower cycle frequency may generate beneficial and adaptive responses in brain, such as ischemic preconditioning ${ }^{28}$. Conversely, chronic, moderate to severe, and high frequency intermittent hypoxia can induce maladaptive disruption of homeostatic mechanisms, leading to dysfunction and sterile neuroinflammation $\underline{17}, \underline{28}$.

Ischemic preconditioning represents a generalized adaptation to ischemia by a variety of cells $\underline{29}, \underline{83}$. In OSA, induction of ischemic preconditioning is thought to be due to the activation of several gene programs, including the hypoxia inducible factor-1, vascular endothelial growth factor, erythropoietin, atrial natriuretic peptide and brain derived neurotrophic factor $\underline{84}, \underline{85}$. Over the years, 
various end-mechanisms and pathways have been shown to play a role, including those of longterm facilitation of phrenic motor output, chemoreflex activation, vascular remodelling, neoangiogenesis, productive autophagy, reactive gliosis, various synaptic alterations, and modulation of adult hippocampal neurogenesis (Figure 4) $17, \underline{86-90}$. CPAP treatment of OSA has been shown to partially reverse the damage in hippocampal regions, and to ameliorate some of the associated cognitive deficits, possibly also by modulating adult neurogenesis $\underline{46}$. In a recent neuroimaging study, co-existence of hypotrophic and hypertrophic changes in brains of OSA patients was taken to reflect the evolving nature of OSA-associated brain injury ${ }^{30}$. It was proposed that any given time ongoing maladaptive neuroinflammatory processes likely exist alongside adaptive mechanisms of increased brain plasticity and ischemic preconditioning $\underline{30}, \underline{83}, \underline{91-93}$. As a corollary to these findings, in a recent study that compared the cognitive performance of patients with high and low hypoxemia after controlling for demographic factors and other aspects of OSA severity, an unexpected advantage of higher hypoxemia on memory was demonstrated in a carefully matched clinical cohort $\underline{94}$.

Another powerful central neuroprotective adaptive mechanism for ischemic events has been demonstrated following the activation of the intrinsic neurons of the cerebellar fastigial nucleus $\stackrel{95}{\text {. }}$ Neurostimulation of these nuclei appears to provide 'protective' reduced excitability of cortical neurons during subsequent ischemic episodes, and to lead to reduced immunoreactivity of cerebral microvessels ${ }^{\underline{2}}$. The 'compensatory' entraining of cerebellum by hypertrophic hippocampi has been proposed to occur in some younger patients with mild OSA $\underline{30}$. Although there are no direct monosynaptic anatomical connections between hippocampi and cerebellum, their connectivity is thought to be important for the control of movement under states of heightened emotion, novel conditions, and for associative learning ${ }^{2}$. Failed adaptation of cerebellar networks to injury has been shown to lead to cognitive deficits and hyperactivity, distractibility, ruminative behaviours, dysphoria and depression in some patients ${ }^{2}, \underline{96}$. Several studies also suggest that, under certain conditions, intermittent hypoxia can increase immune defences without exacerbating inflammation ${ }^{17}, \underline{28}$. Moreover, in animals, short-lasting hypoxic exposures mimicking OSA have been associated with recruitment of bone-marrow derived pluripotent stem cells, which exhibited up-regulation of stem cell differentiation pathways, particularly involving central nervous development and angiogenesis ${ }^{2 \underline{8}}$. The other side of the coin are maladaptive effects of intermittent hypoxia. These include neuroinflammation, and although the exact neurocellular sources for associated processes are still incompletely defined, activation of astroglia is likely to be important $\frac{17}{31}$. In addition, the oligodendrocytes, myelin-producing cells of the CNS, have been shown to be selectively sensitive to hypoxia and sleep fragmentation $\underline{97}, \underline{98}$. The subsequent loss of buffering functions can ultimately contribute to pathological processes, such as increased glial proliferation and microglial activation (Figure $3 b)^{31}$. Astroglial and microglial cells play critical roles in regional blood flow regulation and 
inflammatory processes in the brain, as well as critical coordination of bioenergetics through lactate transport ${ }^{31}$. Under normal conditions, microglia in the healthy CNS exhibit a surveillance phenotype that synthesizes and releases neuroprotective growth/trophic factors ${ }^{31}$. However, severe and prolonged hypoxia can activate microglia toward a toxic, pro-inflammatory phenotype that triggers pathology, including hippocampal apoptosis, impaired synaptic plasticity, and cognitive impairment $\frac{31}{3}$. Neuroinflammation has been shown to independently raise the brain's sensitivity to stress, resulting in stress-related neuropsychiatric disorders, such as anxiety or depression $\underline{\underline{99}}$.

Dynamic changes in transcription of inflammatory genes have been demonstrated following exposure to intermittent hypoxia, with most inflammatory markers increasing over time $\mathrm{e}^{\underline{31}}$. Increased prostaglandin E2 neural tissue concentrations have also been demonstrated in hippocampal and cortical regions accompanied by lipid peroxidation of polyunsaturated fatty acids ${ }^{31}$. Similarly, it has been shown that increased carbonylation and nitrosylation-induced oxidative injury emerges in susceptible brain regions and promotes increased excessive daytime somnolence ${ }^{\underline{17}}, \underline{31}$. Recently, toll-like receptor 4 (TLR4) expression and activity has been demonstrated to be increased on monocytes of patients with OSA $\frac{100}{}$. Similarly, ligands for TLR4 have been shown to be increased in the serum of children with OSA ${ }^{100}$. The microglia of the cortex and brainstem exhibit TLR4 expression after chronic intermittent hypoxia where it is postulated to play region specific and differential (adaptive or maladaptive) role. $\frac{100}{}$ This finding is of particular interest since TLR4 has also been strongly implicated in several inflammatory and neurodegenerative disorders, including vascular dementia and Alzheimer's disease $\frac{100}{}$. In keeping, in cognitively healthy adults, intermittent hypoxia has been correlated with increases in phosphorylated and total tau and amyloid $\beta_{1-42}$ concentrations in cerebral spinal fluid, key components of Alzheimer's pathology 14 . Similarly, cerebral amyloidogenesis and tau phosphorylation along with neuronal degeneration and axonal dysfunction have been demonstrated in cortex and brainstems of animals exposed to intermittent hypoxia ${ }^{15}$. Taken together, these findings support the role for neuroinflammatory processes in cognitive and emotional deficits of OSA patients. They further suggest a close association between hypoxemia induced maladaptive processes and dementia (Figure 4c).

\section{Conclusions and future directions}

The disruption of sleep physiology by OSA is an underappreciated factor, which, together with hypoxemia and other already recognized factors, may further aggravate age-related memory

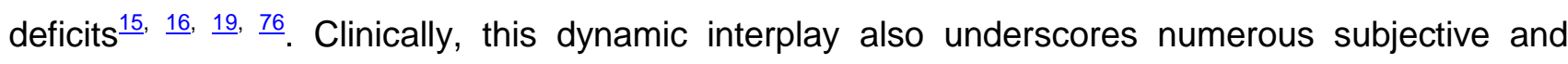
objective cognitive and emotional complaints in some patients $\underline{77}$, $\underline{78}$. An understanding of the proportional impact of these factors, in each individual patient, is a major challenge, because they occur simultaneously and, in all likelihood, target similar neurocircuitry. They also probably share some end-stage adaptive and maladaptive cellular mechanisms ${ }^{1}, \underline{\text { ? }}$. Persistent deficits, even after 
prolonged treatment with CPAP in some patients, suggest that early detection of the CNS sequelae in OSA is vital, so that appropriate treatment is administered before irreversible atrophic and metabolic changes occur. However, the timing and the duration of treatment, and the optimal treatment population, are still unclear and should be addressed in future prospective randomised control studies. Studies reviewed here strongly suggest that tapping into the therapeutic potential of ischemic preconditioning, whilst working on ameliorating the acute and chronic effects of neuroinflammation, may offer legitimate new therapeutical targets in $\mathrm{OSA}^{\underline{14}}, \underline{15}, \underline{17}, \underline{28}, \underline{31}$. Similarly, although they are in their infancy, studies of clinical approaches that target the sleep disturbance arm of this intricate equation advocate a significant future treatment intervention potential ${ }^{14}, \underline{16}, \underline{76}$.

In conclusion, recent findings raise valid questions about the mechanics of associations between OSA and dementia, and further highlight the public health importance of detecting and targeting those at highest risk for cognitive decline.

Contributors: All authors contributed equally to the writing and revision of this manuscript.

The authors do not have any conflicts of interest to report. The authors apologize to all the colleagues whose outstanding work could not be cited due to space limitations.

\section{Search strategy and selection criteria}

We identified references for this Review by searches of PubMed between 2002 and 2015, and references from relevant articles. Additionally, several seminal historic references were added. We used the search terms "sleep apnoea/apnea", "intermittent hypoxia", "sleep", "sleep deprivation/fragmentation", "sleep disordered breathing", and "dementia", "mental health/emotions", "Alzheimer's disease", "brain structure", "cognitive decline", "cognitive function", or "cognition". Articles were restricted to those available in English. We made the final selection by using the most relevant articles.

\section{Key messages}

- Obstructive sleep apnea (OSA) is a prevalent, chronic multisystem disease with a high socioeconomic burden, which remains under diagnosed in the general population.

- OSA is increasingly recognised as one of the potentially modifiable risk factors for dementia; its multiple effects on the central nervous system are acknowledged, albeit their nature and prognosis are yet to be fully understood.

- Both maladaptive and adaptive pathways are likely initiated in the central nervous system during nocturnal apnoeic and hypopnoeic episodes and ensuing sleep fragmentation, the net result of which will depend on the chronicity of process and idiosyncratic characteristics of each patient.

- In future, multimodal investigative approaches should help elucidate more reliable referencing for the acuity of the pathological process, as well as its reversibility following the treatment.

\section{$\underline{\text { References }}$}


1. Gozal D. CrossTalk proposal: The intermittent hypoxia attending severe obstructive sleep apnoea does lead to alterations in brain structure and function. The Journal of physiology 2013; 591(Pt 2): 379-81.

2. Rosenzweig I, Williams SC, Morrell MJ. The impact of sleep and hypoxia on the brain: potential mechanisms for the effects of obstructive sleep apnea. Current opinion in pulmonary medicine 2014; 20(6): 565-71.

3. Young T, Palta M, Dempsey J, Skatrud J, Weber S, Badr S. The occurrence of sleepdisordered breathing among middle-aged adults. The New England journal of medicine 1993; 328(17): 1230-5.

4. Sullivan CE, Issa FG, Berthon-Jones M, Eves L. Reversal of obstructive sleep apnoea by continuous positive airway pressure applied through the nares. Lancet 1981; 1(8225): 862-5.

5. Peppard PE, Young T, Barnet JH, Palta M, Hagen EW, Hla KM. Increased prevalence of sleep-disordered breathing in adults. American journal of epidemiology 2013; 177(9): 1006-14.

6. Young T, Skatrud J, Peppard PE. Risk factors for obstructive sleep apnea in adults. JAMA : the journal of the American Medical Association 2004; 291(16): 2013-6.

7. Sutherland K, Lee RW, Cistulli PA. Obesity and craniofacial structure as risk factors for obstructive sleep apnoea: impact of ethnicity. Respirology 2012; 17(2): 213-22.

8. Ip MS, Lam B, Lauder IJ, et al. A community study of sleep-disordered breathing in middleaged Chinese men in Hong Kong. Chest 2001; 119(1): 62-9.

9. Ip MS, Lam B, Tang LC, Lauder IJ, Ip TY, Lam WK. A community study of sleep-disordered breathing in middle-aged Chinese women in Hong Kong: prevalence and gender differences. Chest 2004; 125(1): 127-34.

10. Tufik S, Santos-Silva R, Taddei JA, Bittencourt LR. Obstructive sleep apnea syndrome in the Sao Paulo Epidemiologic Sleep Study. Sleep medicine 2010; 11(5): 441-6.

11. Li Y, Veasey SC. Neurobiology and neuropathophysiology of obstructive sleep apnea. Neuromolecular medicine 2012; 14(3): 168-79.

12. McNicholas WT, Bonsigore MR, Management Committee of ECAB. Sleep apnoea as an independent risk factor for cardiovascular disease: current evidence, basic mechanisms and research priorities. The European respiratory journal : official journal of the European Society for Clinical Respiratory Physiology 2007; 29(1): 156-78.

13. Jordan AS, McSharry DG, Malhotra A. Adult obstructive sleep apnoea. Lancet 2014; 383(9918): 736-47.

14. Yaffe K, Falvey CM, Hoang T. Connections between sleep and cognition in older adults. Lancet neurology 2014; 13(10): 1017-28.

15. Daulatzai MA. Death by a thousand cuts in Alzheimer's disease: hypoxia--the prodrome. Neurotoxicity research 2013; 24(2): 216-43.

16. Pan W, Kastin AJ. Can sleep apnea cause Alzheimer's disease? Neuroscience and biobehavioral reviews 2014; 47C: 656-69.

17. Lavie L. Oxidative stress in obstructive sleep apnea and intermittent hypoxia - Revisited The bad ugly and good: Implications to the heart and brain. Sleep medicine reviews 2014.

18. Poe GR, Walsh CM, Bjorness TE. Cognitive neuroscience of sleep. Progress in brain research 2010; 185: 1-19.

19. Mander BA, Rao V, Lu B, et al. Prefrontal atrophy, disrupted NREM slow waves and impaired hippocampal-dependent memory in aging. Nature neuroscience 2013; 16(3): 357-64.

20. Engleman $\mathrm{H}$, Joffe D. Neuropsychological function in obstructive sleep apnoea. Sleep medicine reviews 1999; 3(1): 59-78.

21. Marshall NS, Barnes M, Travier N, et al. Continuous positive airway pressure reduces daytime sleepiness in mild to moderate obstructive sleep apnoea: a meta-analysis. Thorax 2006; 61(5): 430-4.

22. Olaithe M, Skinner TC, Hillman D, Eastwood PE, Bucks RS. Cognition and nocturnal disturbance in OSA: the importance of accounting for age and premorbid intelligence. Sleep \& breathing = Schlaf \& Atmung 2014.

23. Bucks RS, Olaithe M, Eastwood P. Neurocognitive function in obstructive sleep apnoea: a meta-review. Respirology 2013; 18(1): 61-70. 
24. McMillan A, Bratton, D.J., Faria, R., Laskawiec-Szkonter, M., Griffin, S., Davies, R.J., Nunn, A.J., Stradling, J.R., Riha, R.L., Morrell, M.J. A 12 month multicentre, randomised trial of Continuous Positive Airway Pressure in older people with Obstructive Sleep Apnea Syndrome. Lancet Resp Medicine 2014.

25. Craig SE, Kohler M, Nicoll D, et al. Continuous positive airway pressure improves sleepiness but not calculated vascular risk in patients with minimally symptomatic obstructive sleep apnoea: the MOSAIC randomised controlled trial. Thorax 2012; 67(12): 1090-6.

26. Hajak G, Klingelhofer J, Schulz-Varszegi M, Sander D, Ruther E. Sleep apnea syndrome and cerebral hemodynamics. Chest 1996; 110(3): 670-9.

27. Rosenzweig I, Williams SC, Morrell MJ. CrossTalk opposing view: the intermittent hypoxia attending severe obstructive sleep apnoea does not lead to alterations in brain structure and function. The Journal of physiology 2013; 591(Pt 2): 383-5; discussion 7,9.

28. Almendros I, Wang Y, Gozal D. The polymorphic and contradictory aspects of intermittent hypoxia. American journal of physiology Lung cellular and molecular physiology 2014; 307(2): L12940.

29. Lavie L, Lavie P. Ischemic preconditioning as a possible explanation for the age decline relative mortality in sleep apnea. Medical hypotheses 2006; 66(6): 1069-73.

30. Rosenzweig I, Kempton MJ, Crum WR, et al. Hippocampal hypertrophy and sleep apnea: a role for the ischemic preconditioning? PloS one 2013; 8(12): e83173.

31. Dale EA, Ben Mabrouk F, Mitchell GS. Unexpected benefits of intermittent hypoxia: enhanced respiratory and nonrespiratory motor function. Physiology 2014; 29(1): 39-48.

32. Vaessen TJ, Overeem S, Sitskoorn MM. Cognitive complaints in obstructive sleep apnea. Sleep medicine reviews 2014.

33. Pullens MJ, De Vries J, Roukema JA. Subjective cognitive dysfunction in breast cancer patients: a systematic review. Psycho-oncology 2010; 19(11): 1127-38.

34. Giles TL, Lasserson TJ, Smith BH, White J, Wright J, Cates CJ. Continuous positive airways pressure for obstructive sleep apnoea in adults. The Cochrane database of systematic reviews 2006; (3): CD001106.

35. Kylstra WA, Aaronson JA, Hofman WF, Schmand BA. Neuropsychological functioning after CPAP treatment in obstructive sleep apnea: a meta-analysis. Sleep medicine reviews 2013; 17(5): 341-7.

36. Antic NA, Catcheside P, Buchan $\mathrm{C}$, et al. The effect of CPAP in normalizing daytime sleepiness, quality of life, and neurocognitive function in patients with moderate to severe OSA. Sleep 2011; 34(1): 111-9.

37. Weaver TE, Maislin G, Dinges DF, et al. Relationship between hours of CPAP use and achieving normal levels of sleepiness and daily functioning. Sleep 2007; 30(6): 711-9.

38. Castronovo V, Scifo P, Castellano A, et al. White matter integrity in obstructive sleep apnea before and after treatment. Sleep 2014; 37(9): 1465-75.

39. Troussiere AC, Charley CM, Salleron J, et al. Treatment of sleep apnoea syndrome decreases cognitive decline in patients with Alzheimer's disease. Journal of neurology, neurosurgery, and psychiatry 2014; 85(12): 1405-8.

40. Mason M, Welsh EJ, Smith I. Drug therapy for obstructive sleep apnoea in adults. The Cochrane database of systematic reviews 2013; 5: CD003002.

41. Kohler M, Bloch KE, Stradling JR. Pharmacological approaches to the treatment of obstructive sleep apnoea. Expert opinion on investigational drugs 2009; 18(5): 647-56.

42. Twigg GL, Papaioannou I, Jackson M, et al. Obstructive sleep apnea syndrome is associated with deficits in verbal but not visual memory. American journal of respiratory and critical care medicine 2010; 182(1): 98-103.

43. Kilpinen $\mathrm{R}$, Saunamaki $\mathrm{T}$, Jehkonen M. Information processing speed in obstructive sleep apnea syndrome: a review. Acta neurologica Scandinavica 2014; 129(4): 209-18.

44. Andreou G, Vlachos F, Makanikas K. Effects of chronic obstructive pulmonary disease and obstructive sleep apnea on cognitive functions: evidence for a common nature. Sleep disorders 2014; 2014: 768210. 
45. Andreou G, Agapitou P. Reduced language abilities in adolescents who snore. Archives of clinical neuropsychology : the official journal of the National Academy of Neuropsychologists 2007; 22(2): 225-9.

46. Canessa N, Castronovo V, Cappa SF, et al. Obstructive sleep apnea: brain structural changes and neurocognitive function before and after treatment. American journal of respiratory and critical care medicine 2011; 183(10): 1419-26.

47. Prilipko O, Huynh N, Schwartz S, et al. The effects of CPAP treatment on task positive and default mode networks in obstructive sleep apnea patients: an fMRI study. PloS one 2012; 7(12): e47433.

48. Ferini-Strambi L, Marelli S, Galbiati A, Castronovo C. Effects of continuous positive airway pressure on cognitition and neuroimaging data in sleep apnea. International journal of psychophysiology : official journal of the International Organization of Psychophysiology 2013; 89(2): 203-12.

49. Kushida CA, Nichols DA, Holmes TH, et al. Effects of continuous positive airway pressure on neurocognitive function in obstructive sleep apnea patients: The Apnea Positive Pressure Longterm Efficacy Study (APPLES). Sleep 2012; 35(12): 1593-602.

50. Biggs SN, Vlahandonis A, Anderson V, et al. Long-term changes in neurocognition and behavior following treatment of sleep disordered breathing in school-aged children. Sleep 2014; 37(1): 77-84.

51. Gozal D. Sleep-disordered breathing and school performance in children. Pediatrics 1998; 102(3 Pt 1): 616-20.

52. Cattell RB. The theory of fluid and crystallized general intelligence checked at the 5-6 yearold level. The British journal of educational psychology 1967; 37(2): 209-24.

53. Biggs SN, Walter LM, Nisbet LC, et al. Time course of EEG slow-wave activity in pre-school children with sleep disordered breathing: a possible mechanism for daytime deficits? Sleep medicine 2012; 13(8): 999-1005.

54. Vyazovskiy VV, Delogu A. NREM and REM Sleep: Complementary Roles in Recovery after Wakefulness. The Neuroscientist : a review journal bringing neurobiology, neurology and psychiatry 2014; 20(3): 203-19.

55. Neubauer AC, Fink A. Intelligence and neural efficiency. Neuroscience and biobehavioral reviews 2009; 33(7): 1004-23.

56. Tononi G, Cirelli C. Sleep and the price of plasticity: from synaptic and cellular homeostasis to memory consolidation and integration. Neuron 2014; 81(1): 12-34.

57. Abel T, Havekes R, Saletin JM, Walker MP. Sleep, plasticity and memory from molecules to whole-brain networks. Current biology : CB 2013; 23(17): R774-88.

58. Picchioni D, Reith RM, Nadel JL, Smith CB. Sleep, plasticity and the pathophysiology of neurodevelopmental disorders: the potential roles of protein synthesis and other cellular processes. Brain sciences 2014; 4(1): 150-201.

59. Franco CM, Lima AM, Ataide L, Jr., et al. Obstructive sleep apnea severity correlates with cellular and plasma oxidative stress parameters and affective symptoms. Journal of molecular neuroscience : MN 2012; 47(2): 300-10.

60. Wong KK, Marshall NS, Grunstein RR, Dodd MJ, Rogers NL. Comparing the neurocognitive effects of $40 \mathrm{~h}$ sustained wakefulness in patients with untreated OSA and healthy controls. Journal of sleep research 2008; $17(3)$ : 322-30.

61. D'Rozario AL, Kim JW, Wong KK, et al. A new EEG biomarker of neurobehavioural impairment and sleepiness in sleep apnea patients and controls during extended wakefulness. Clinical neurophysiology : official journal of the International Federation of Clinical Neurophysiology 2013; 124(8): 1605-14.

62. Vakulin A, Baulk SD, Catcheside PG, et al. Effects of alcohol and sleep restriction on simulated driving performance in untreated patients with obstructive sleep apnea. Annals of internal medicine 2009; 151(7): 447-55.

63. Vakulin A, Catcheside PG, Baulk SD, et al. Individual variability and predictors of driving simulator impairment in patients with obstructive sleep apnea. Journal of clinical sleep medicine : JCSM : official publication of the American Academy of Sleep Medicine 2014; 10(6): 647-55. 
64. Killgore WD. Effects of sleep deprivation on cognition. Progress in brain research 2010; 185: 105-29.

65. Gelber RP, Redline S, Ross GW, et al. Associations of brain lesions at autopsy with polysomnography features before death. Neurology 2014.

66. Cipolli C, Mazzetti M, Plazzi G. Sleep-dependent memory consolidation in patients with sleep disorders. Sleep medicine reviews 2013; 17(2): 91-103.

67. Schonwald SV, Carvalho DZ, de Santa-Helena EL, Lemke N, Gerhardt GJ. Topographyspecific spindle frequency changes in obstructive sleep apnea. BMC neuroscience 2012; 13: 89.

68. Carvalho DZ, Gerhardt GJ, Dellagustin G, et al. Loss of sleep spindle frequency deceleration in Obstructive Sleep Apnea. Clinical neurophysiology : official journal of the International Federation of Clinical Neurophysiology 2014; 125(2): 306-12.

69. Ondze B, Espa F, Dauvilliers Y, Billiard M, Besset A. Sleep architecture, slow wave activity and sleep spindles in mild sleep disordered breathing. Clinical neurophysiology : official journal of the International Federation of Clinical Neurophysiology 2003; 114(5): 867-74.

70. Landmann N, Kuhn M, Piosczyk H, et al. The reorganisation of memory during sleep. Sleep medicine reviews 2014; 18(6): 531-41.

71. Morisson F, Lavigne G, Petit D, Nielsen T, Malo J, Montplaisir J. Spectral analysis of wakefulness and REM sleep EEG in patients with sleep apnoea syndrome. The European respiratory journal : official journal of the European Society for Clinical Respiratory Physiology 1998; 11(5): 1135-40.

72. Daurat A, Foret J, Bret-Dibat JL, Fureix C, Tiberge M. Spatial and temporal memories are affected by sleep fragmentation in obstructive sleep apnea syndrome. Journal of clinical and experimental neuropsychology 2008; 30(1): 91-101.

73. Mokhlesi B, Finn LA, Hagen EW, et al. Obstructive Sleep Apnea during REM Sleep and Hypertension. Results of the Wisconsin Sleep Cohort. American journal of respiratory and critical care medicine 2014; 190(10): 1158-67.

74. Mokhlesi B, Punjabi NM. "REM-related" obstructive sleep apnea: an epiphenomenon or a clinically important entity? Sleep 2012; 35(1): 5-7.

75. Varga AW, Kishi A, Mantua J, et al. Apnea-induced rapid eye movement sleep disruption impairs human spatial navigational memory. The Journal of neuroscience : the official journal of the Society for Neuroscience 2014; 34(44): 14571-7.

76. Mander BA, Rao V, Lu B, et al. Impaired prefrontal sleep spindle regulation of hippocampaldependent learning in older adults. Cerebral cortex 2014; 24(12): 3301-9.

77. Sforza E, Roche F. Sleep apnea syndrome and cognition. Frontiers in neurology 2012; 3: 87. 78. Goldstein AN, Walker MP. The role of sleep in emotional brain function. Annual review of clinical psychology 2014; 10: 679-708.

79. Povitz M, Bolo CE, Heitman SJ, Tsai WH, Wang J, James MT. Effect of treatment of obstructive sleep apnea on depressive symptoms: systematic review and meta-analysis. PLoS medicine 2014; 11(11): e1001762.

80. Gupta MA, Simpson FC. Obstructive Sleep Apnea and Psychiatric Disorders: A Systematic Review. Journal of clinical sleep medicine : JCSM : official publication of the American Academy of Sleep Medicine 2014.

81. Sharafkhaneh A, Giray N, Richardson P, Young T, Hirshkowitz M. Association of psychiatric disorders and sleep apnea in a large cohort. Sleep 2005; 28(11): 1405-11.

82. Ayalon L, Ancoli-Israel S, Drummond SP. Obstructive sleep apnea and age: a double insult to brain function? American journal of respiratory and critical care medicine 2010; 182(3): 413-9.

83. Dirnagl U, Becker K, Meisel A. Preconditioning and tolerance against cerebral ischaemia: from experimental strategies to clinical use. Lancet neurology 2009; 8(4): 398-412.

84. Nanduri J, Yuan G, Kumar GK, Semenza GL, Prabhakar NR. Transcriptional responses to intermittent hypoxia. Respiratory physiology \& neurobiology 2008; 164(1-2): 277-81.

85. Brzecka A. Brain preconditioning and obstructive sleep apnea syndrome. Acta neurobiologiae experimentalis 2005; 65(2): 213-20.

86. Haddad GG, Yu SP. Brain hypoxia and ischemia : with special emphasis on development. New York: Humana Press; 2009. 
87. Papadakis M, Hadley G, Xilouri M, et al. Tsc1 (hamartin) confers neuroprotection against ischemia by inducing autophagy. Nature medicine 2013; 19(3): 351-7.

88. Tsai YW, Yang YR, Sun SH, Liang KC, Wang RY. Post ischemia intermittent hypoxia induces hippocampal neurogenesis and synaptic alterations and alleviates long-term memory impairment. Journal of cerebral blood flow and metabolism : official journal of the International Society of Cerebral Blood Flow and Metabolism 2013.

89. Tsai YW, Yang YR, Wang PS, Wang RY. Intermittent hypoxia after transient focal ischemia induces hippocampal neurogenesis and c-Fos expression and reverses spatial memory deficits in rats. PloS one $2011 ; 6(8)$ : e24001.

90. Aviles-Reyes RX, Angelo MF, Villarreal A, Rios H, Lazarowski A, Ramos AJ. Intermittent hypoxia during sleep induces reactive gliosis and limited neuronal death in rats: implications for sleep apnea. Journal of neurochemistry 2010; 112(4): 854-69.

91. Seki T. Neurogenesis in the adult brain. Tokyo ; New York: Springer; 2011.

92. Lledo PM, Alonso M, Grubb MS. Adult neurogenesis and functional plasticity in neuronal circuits. Nature reviews Neuroscience 2006; 7(3): 179-93.

93. Ferriero DM. Protecting neurons. Epilepsia 2005; 46 Suppl 7: 45-51.

94. Hoth KF, Zimmerman ME, Meschede KA, Arnedt JT, Aloia MS. Obstructive sleep apnea: impact of hypoxemia on memory. Sleep \& breathing = Schlaf \& Atmung 2013; 17(2): 811-7.

95. Reis DJ, Golanov EV, Galea E, Feinstein DL. Central neurogenic neuroprotection: central neural systems that protect the brain from hypoxia and ischemia. Annals of the New York Academy of Sciences 1997; 835: 168-86.

96. Schmahmann JD, Sherman JC. The cerebellar cognitive affective syndrome. Brain : a journal of neurology 1998; 121 ( Pt 4): 561-79.

97. Rosenzweig I, Vukadinovic Z, Turner AJ, Catani M. Neuroconnectivity and valproic acid: the myelin hypothesis. Neuroscience and biobehavioral reviews 2012; 36(8): 1848-56.

98. Bellesi M, Pfister-Genskow M, Maret S, Keles S, Tononi G, Cirelli C. Effects of sleep and wake on oligodendrocytes and their precursors. The Journal of neuroscience : the official journal of the Society for Neuroscience 2013; 33(36): 14288-300.

99. Skaper SD, Facci L, Giusti P. Neuroinflammation, Microglia and Mast Cells in the Pathophysiology of Neurocognitive Disorders: A Review. CNS \& neurological disorders drug targets 2014.

100. Unnikrishnan D, Jun J, Polotsky V. Inflammation in sleep apnea: An update. Reviews in endocrine \& metabolic disorders 2014.

101. Desseilles M, Dang-Vu T, Schabus M, Sterpenich V, Maquet P, Schwartz S. Neuroimaging insights into the pathophysiology of sleep disorders. Sleep 2008; 31(6): 777-94.

102. Dingli K, Assimakopoulos T, Fietze I, Witt C, Wraith PK, Douglas NJ. Electroencephalographic spectral analysis: detection of cortical activity changes in sleep apnoea patients. The European respiratory journal : official journal of the European Society for Clinical Respiratory Physiology 2002; 20(5): 1246-53.

103. Morisson F, Decary A, Petit D, Lavigne G, Malo J, Montplaisir J. Daytime sleepiness and EEG spectral analysis in apneic patients before and after treatment with continuous positive airway pressure. Chest 2001; 119(1): 45-52.

104. Metzler-Baddeley C, Hunt S, Jones DK, Leemans A, Aggleton JP, O'Sullivan MJ. Temporal association tracts and the breakdown of episodic memory in mild cognitive impairment. Neurology 2012; 79(23): 2233-40.

105. Macey PM, Kumar R, Woo MA, Valladares EM, Yan-Go FL, Harper RM. Brain structural changes in obstructive sleep apnea. Sleep 2008; 31(7): 967-77.

106. Small SA, Schobel SA, Buxton RB, Witter MP, Barnes CA. A pathophysiological framework of hippocampal dysfunction in ageing and disease. Nature reviews Neuroscience 2011; 12(10): 585601. 
Figure 1: Brain regions and mechanisms involved in sleep apnoea injury. The nocturnal episodes of complete or partial pharyngeal obstruction result in intermittent hypoxia and sleep fragmentation. Both intermittent hypoxia and sleep fragmentation can aggravate brain injury (red arrows) and cause hypotrophic changes in several brain regions shown. Ensuing neurophysiological and neurochemical changes can also manifest in cognitive and emotional deficits that can be ameliorated (white arrows), using treatment with the continuous positive airway pressure therapy (CPAP) and or ischaemic preconditioning. Adapted from $\underline{101}$

Figure 2: The role for sleep in cognition and emotions. (a) Cognitive Sleep: sleep apnoea and ageing can independently cause grey matter atrophy in the prefrontal cortex. Atrophy can mediate the degree of slow wave activity (SWA) disruption, whilst SWA in turn can mediate the degree of impaired memory retention ${ }^{19}$. SWA activity disruption likely also leads to cellular stress ${ }^{58}$. (b) Emotional Sleep: Conceptual schematics of "the sleep to forget and sleep to remember" model are shown ${ }^{78}$. Over one or several nights and numerous repetitions of this REM mechanism, sleep transforms an emotional memory into a memory of an emotional event that is no longer emotional. Reproduced with permission and adapted $\underline{58}$ from $\underline{19}, \underline{58}, \underline{78}$.

Figure 3: Adaptive and maladaptive processes induced by intermittent hypoxia. Conceptual presentation of the net effect of cycles of intermittent hypoxia, of varied length and frequency, over a period of time (minutes to days to weeks) (a). High doses still elicit neuroadaptive mechanisms, however the balance is shifted and maladaptive processes such as (b) neuroinflammation are likely to be instigated. Finding an optimal dose is key to developing effective treatment. Reproduced with permission from ${ }^{31}$. (c) Possible cascade of interactions between sleep apnoea and Alzheimer's disease.

Figure 4: Putative theoretical neuromechanisms behind plastic changes noted in clinical studies of sleep apnoea patients.

Theta $(\theta)$ is the archetypical rhythmic activity in the hippocampus, which runs from the dentate gyrus

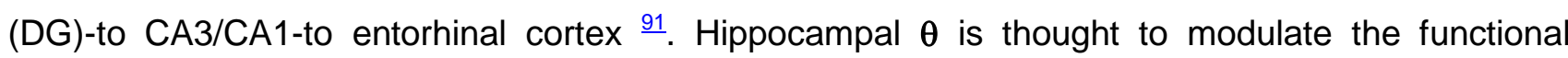

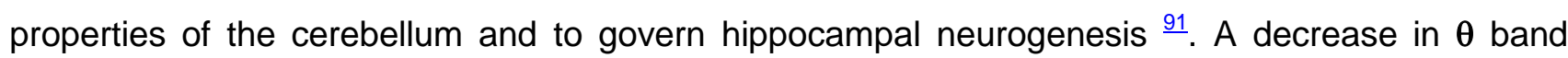

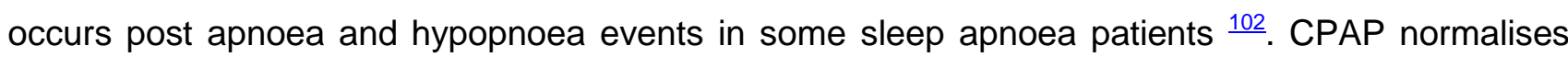

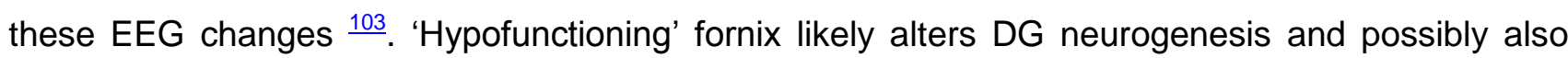
contributes to mild cognitive decline in OSA $\frac{104}{105}$. Reproduced with permission from $106, ?$. 